\title{
O MÉTODO DE REGIÃO DE CONFIANÇA DE BYRD-OMOJOKUN APLICADO AO FLUXO DE POTÊNCIA ÓTIMO
}

\author{
Andréa A. Sousa* \\ tnandrea@yahoo.com
}

\author{
Geraldo L. Torres* \\ gltorres@ufpe.br
}

*Departamento de Engenharia Elétrica e Sistemas de Potência

Universidade Federal de Pernambuco

\begin{abstract}
Robust Optimal Power Flow by Byrd-Omojukun Trust Region Method

This paper presents a globally convergent Optimal Power Flow (OPF) algorithm, i.e., an optimization algorithm capable of finding an OPF solution whenever a solution exists. As power systems become heavily loaded and operate close to security limits there is an increasing need for globally convergent OPF algorithms. The proposed algorithm uses the trust region technique of Byrd and Omojokun with the trust region constraint defined by the infinity norm. The subproblems generated in each trust region iteration are solved by primal-dual interior-point methods for quadratic programming. The focus of the proposed OPF algorithm is on the convergence robustness rather than on processing time. The simulations results with the IEEE test systems suggest the expected robustness of the approach.
\end{abstract}

KEYWORDS: Optimal Power Flow, Trust-Region Methods, Interior-Point Methods, Global Convergence.

\section{RESUMO}

Este artigo apresenta um algoritmo de Fluxo de Potência Ótimo (FPO) globalmente convergente, ou seja, capaz de obter uma solução de FPO sempre que ela existir. Como os sistemas de potência estão operando cada vez mais próximos dos limites de segurança, há uma necessi-

Artigo submetido em 08/01/2009 (Id.: 00939)

Revisado em 30/04/2009, 23/11/2009

Aceito sob recomendação do Editor Associado Prof. Alexandre Bazanella dade crescente por algoritmos de FPO com convergência global. O algoritmo proposto utiliza a técnica de região de confiança de Byrd e Omojokun, mas com a restrição de região de confiança definida pela norma infinita. Os subproblemas gerados em cada iteração de região de confiança são resolvidos por métodos de pontos-interiores para programação quadrática. O foco do novo algoritmo de FPO está na robustez de convergência e não no tempo de processamento. Os resultados de simulações com os sistemas testes do IEEE sugerem a esperada robustez de convergência do algoritmo.

PalaVRaS-Chave: Fluxo de Potência Ótimo, Métodos de Região de Confiança, Métodos de Pontos-Interiores, Convergência Global.

\section{INTRODUÇÃO}

Na indústria de potência atual a tendência natural é que os sistemas elétricos de potência operem próximos aos seus limites de segurança, dando origem a fortes nãolinearidades no ponto de solução dos modelos matemáticos dos sistemas, tais como modelos de Fluxo de Potência Ótimo (FPO). A reestruturação do setor elétrico tem resultado também em novos e complexos modelos de FPO (Wang et al., 2007), desafiando as técnicas de solução comumente empregadas, principalmente no que diz respeito a robustez de convergência. Neste aspecto, este artigo apresenta um novo algoritmo de FPO com a característica de convergência global.

Por convergência global entende-se que o algoritmo de otimização deve ser capaz de obter uma solução, se ao 
menos uma existir, com qualquer escolha do ponto inicial. Há duas abordagens clássicas para a globalização de um algoritmo localmente convergente (Nocedal e Wright, 1999): utilizar uma busca em linha ou impor uma região de confiança. Neste artigo, considera-se um método de região de confiança devido ao grande sucesso que esses métodos têm alcançado na globalização de algoritmos para otimização irrestrita (Plantenga, 1994). Mais especificamente, o artigo descreve uma aplicação no problema de FPO não-linear da técnica de região de confiança proposta por Byrd (Byrd, 1987) e Omojokun (Omojokun, 1989), devido a eficiência dessa técnica no tratamento de inconsistência das restrições.

Os métodos de região de confiança constituem uma classe relativamente nova de algoritmos de otimização que minimizam uma aproximação quadrática de uma função objetivo não-linear dentro de uma região fechada chamada de região de confiança. Essa região fechada é assim denominada porque dentro dela o modelo quadrático pode ser confiado como uma boa aproximação para a função objetivo não-linear original. Os métodos de região de confiança diferem entre si na forma que modelam a função objetivo e tratam as restrições (Nocedal e Wright, 1999; Conn et al., 2000), principalmente as restrições de desigualdades. A técnica de Byrd e Omojokun estudada neste artigo pode ser vista como uma Programação Quadrática Sequencial (PQS) com uma região de confiança. Ela decompõe cada subproblema de programação quadrática em dois subproblemas menores de solução mais fácil, tornando a técnica bastante atrativa para otimização de grande escala, como ocorre nos problemas de FPO.

Algumas aplicações de métodos de região de confiança em sistemas de potência são encontradas na literatura. Pajic e Clements (Pajic e Clements, 2003) foram os primeiros a utilizar um algoritmo de região de confiança para resolver problemas de estimação de estados. Costa, Salgado e Haas (Costa et al., 2007) também descrevem uma aplicação de métodos de região de confiança em problemas de estimação de estados. Wang et al (Wang et al., 2007) aplicam um algoritmo de região de confiança em modelos de FPO de mercado de energia envolvendo funções não-suaves (funções não continuamente diferenciáveis). Sousa e Torres (Sousa e Torres, 2007) apresentam um algoritmo de pontos-interiores com região de confiança para solução do FPO não-linear.

Algoritmos de otimização globalmente convergentes são invariavelmente computacionamente onerosos. Código complexo e tempo de processamento elevado são o preço pago por robustez de convergência. Para contornar tais dificuldades, neste artigo associa-se uma técnica de região de confiança com métodos de pontos-interiores. Esta associação pode ser de duas formas: (i) aplicar um método de região de confiança na solução do problema de barreira logaritmica para globalizar a solução, ou (ii) aplicar um método de pontos-interiores para resolver os subproblemas de região de confiança. A abordagem (ii) é a utilizada aqui. Primeiro, a técnica de Byrd e Omojokun decompõe cada subproblema de região de confiança em dois subproblemas menores de solução mais fácil. A seguir, um algoritmo primal-dual de pontos-interiores é utilizado para resolver os subproblemas de PQ gerados.

Este artigo avança no desenvolvimento do algoritmo de FPO em (Sousa e Torres, 2007), em especial na solução dos subproblemas de região de confiança e atualização dos parâmetros. Os subproblemas vertical e horizontal gerados pela técnica de Byrd e Omojokun são expressos numa forma padrão comum e resolvidos por um único algoritmo de pontos-interiores formatado para essa forma padrão. Diversas técnicas de solução dos subproblemas de região de confiança são descritas na literatura (Moré, 1983; Sorensen, 1982). Todavia, a solução via métodos de pontos-interiores usada neste artigo permite o interfaceamento do algoritmo proposto com algoritmos de FPO baseados em métodos de pontos-interiores, o que vem a ser uma característica interessante. Muitas aplicações práticas de FPO requerem algoritmos rápidos de otimização, e métodos de pontos-interiores geralmente atendem esse requisito. Entretanto, falha de convergência em alguns casos por ser um grande problema (Almeida, 2006). Sendo mais robusto porém mais lento, um algoritmo de região de confiança não tem como propósito ser a técnica de resolução do FPO em todas as circunstâncias, mas uma técnica complementar aos algoritmos mais rápidos mas menos robustos.

A organização do restante do texto é como segue. Na Seção 2 é apresentado o desenvolvimento detalhado do algoritmo de região de confiança, com base na técnica de Byrd e Omojokun, para resolver uma forma padrão do problema de FPO contendo restrições de igualdades não-lineares e restrições de limites simples sobre as variáveis. Na Seção 3 o algoritmo de pontos-interiores para resolver os subproblemas de região de confiança é descrito brevemente. Na Seção 4 são discutidos alguns aspectos da implementação computacional e na Seção 5 são apresentados e analisados os resultados numéricos. A Seção 6 conclui o artigo com as observações finais. 


\section{A TÉCNICA DE REGIÃO DE CONFIAN- ÇA DE BYRD E OMOJOKUN}

A técnica de região de confiança descrita nesta seção considera problemas de FPO na forma:

$$
\begin{aligned}
\min & f(x) \\
\text { sujeito a } & g(x)=0 \\
& \underline{x} \leq x \leq \bar{x}
\end{aligned}
$$

em que $x \in \mathbb{R}^{n}$ é o vetor de variáveis de decisão, incluindo as variáveis de controle e de estado; $g: \mathbb{R}^{n} \rightarrow$ $\mathbb{R}^{m}$ é um vetor de funções não-lineares com as equações de fluxo de potência convencionais e outras restrições de igualdade; e $\underline{x}$ e $\bar{x}$ são limites inferiores e superiores sobre as variáveis $x$, correspondendo a limites físicos $\mathrm{e}$ operacionais sobre o sistema.

Para resolver (1), um método de região de confiança geralmente considera uma aproximação de PQ (objetivo quadrático e restrições lineares) estendida por uma restrição de região de confiança, como segue:

$$
\begin{aligned}
\min & f\left(x_{k}\right)+\nabla f\left(x_{k}\right)^{T} d+\frac{1}{2} d^{T} H_{k} d \\
\text { sujeito a } & g\left(x_{k}\right)+\nabla g\left(x_{k}\right)^{T} d=0 \\
& \underline{x} \leq x_{k}+d \leq \bar{x} \\
& \|d\| \leq \Delta_{k}
\end{aligned}
$$

em que $\Delta_{k}$ é o raio da região de confiança, $\nabla f(x) \in \mathbb{R}^{n}$ é o gradiente de $f(x), \nabla g\left(x_{k}\right) \in \mathbb{R}^{n \times m}$ é a matriz de gradientes de $g(x)$, e $H_{k} \in \mathbb{R}^{n \times n}$ é a Hessiana da função de Lagrange associada a (1), calculada por:

$$
H_{k}=\nabla^{2} f\left(x_{k}\right)+\sum_{i=1}^{m} \lambda_{i}^{k} \nabla^{2} g_{i}\left(x_{k}\right),
$$

em que $\nabla^{2} g_{i}\left(x_{k}\right) \in \mathbb{R}^{n \times n}$ é a Hessiana da função de restrição $g_{i}(x)$ e $\lambda_{i}$ é o multiplicador de Lagrange associado à restrição.

Baseado em (Gomes et al., 1999), a restrição de região de confiança (2d) é aqui definida usando a norma infinita, ou seja, $\|d\|_{\infty} \leq \Delta_{k}$, gerando subproblemas de região de confiança do tipo programação quadrática, de solução mais fácil do que utilizando a norma Euclideana. Uma vez que $\|d\|_{\infty}=\max _{i}\left|d_{i}\right|$, as restrições (2c) e (2d) sobre o passo $d$ podem ser combinadas numa única restrição, e o subproblema pode ser reescrito como:

$$
\begin{aligned}
\min & f\left(x_{k}\right)+\nabla f\left(x_{k}\right)^{T} d+\frac{1}{2} d^{T} H_{k} d \\
\text { sujeito a } & g\left(x_{k}\right)+\nabla g\left(x_{k}\right)^{T} d=0 \\
& \max \left\{\underline{\delta},-\Delta_{k}\right\} \leq d \leq \min \left\{\bar{\delta}, \Delta_{k}\right\}
\end{aligned}
$$

em que $\underline{\delta}=\underline{x}-x_{k}$ e $\bar{\delta}=\bar{x}-x_{k}$. Como a restrição (2d) utiliza a norma infinita, as restrições em (4) são lineares e o subproblema de região de confiança é tipo PQ. Independente da norma utilizada, é possível que a restrição de região de confiança (4c) restrinja o tamanho do passo $d$ de forma tal que as restrições (4b) tornem-se incompatíveis (Nocedal e Wright, 1999). Ou seja, um passo $d$ satisfazendo (4b) pode não interceptar a região de confiança (4c). Esse problema não pode ser evitado aumentando o raio da região de confiança, uma vez que a convergência global do método depende da capacidade dele reduzir $\Delta_{k}$ até que o subproblema aproxime com precisão o problema original. Portanto, restrições incompatíveis podem ser inevitáveis em um subproblema de região de confiança.

Para resolver o possível conflito no atendimento de (4b) e (4c), na técnica de Byrd-Omojokun a solução de (4) é dividida em dois subproblemas menores, denominados de subproblemas vertical e horizontal. É essa habilidade de lidar com restrições linearizadas incompatíveis que torna o método de região de confiança globalmente convergente, ao contrário de outros métodos populares baseados em aproximações sucessivas, tais como programação linear e quadrática sequencial.

O subproblema vertical é definido como:

$$
\begin{aligned}
\min & \left\|g\left(x_{k}\right)+\nabla g\left(x_{k}\right)^{T} v\right\|_{2}^{2} \\
\text { sujeito a } & \max \left\{\underline{\delta},-\xi \Delta_{k}\right\} \leq v \leq \min \left\{\bar{\delta}, \xi \Delta_{k}\right\}
\end{aligned}
$$

em que $\xi \in(0,1)$ é um fator de contração que define uma região de confiança reduzida (tipicamente, $\xi=0.8$ ). Desprezando-se o termo constante na função objetivo quadrática, o subproblema (5) pode ser reescrito como

$$
\begin{array}{ll}
\min & \left(\nabla g\left(x_{k}\right) g\left(x_{k}\right)\right)^{T} v+\frac{1}{2} v^{T} \nabla g\left(x_{k}\right) \nabla g\left(x_{k}\right)^{T} v \\
\text { s. a } & \max \left\{\underline{\delta},-\xi \Delta_{k}\right\} \leq v \leq \min \left\{\bar{\delta}, \xi \Delta_{k}\right\}
\end{array}
$$

A finalidade do subproblema vertical (5) é encontrar um passo vertical $v$ que localiza-se bem no interior da região de confiança (de acordo com o fator de contração $\xi$ ) e que procura satisfazer as restrições de igualdades (4b) o máximo possível (minimizando o quadrado da norma-2 dos resíduos das restrições). Claramente, o subproblema vertical envolve a minimização de uma função quadrática convexa com restrições de caixa, de forma que ele tem uma solução a qual pode ser obtida por métodos bastante conhecidos para este tipo de problema.

Obtido o passo vertical $v_{k}$, o passo completo $d_{k}$ é obtido 
resolvendo-se o subproblema horizontal:

$$
\begin{aligned}
\min & \nabla f\left(x_{k}\right)^{T} d+\frac{1}{2} d^{T} H_{k} d \\
\text { sujeito a } & \nabla g\left(x_{k}\right)^{T} d=\nabla g\left(x_{k}\right)^{T} v_{k} \\
& \max \left\{\underline{\delta},-\Delta_{k}\right\} \leq v \leq \min \left\{\bar{\delta}, \Delta_{k}\right\} .
\end{aligned}
$$

A restrição de igualdade (7b) é uma relaxação de (4b) de forma que (7b) e (7c) são sempre consistentes; por exemplo, $d=v_{k}$ é viável. No entanto, é o subproblema vertical que relaxa a restrição original o suficiente para permitir consistência. A restrição (7b) tem o efeito de forçar o passo final $d_{k}$ a fazer o mesmo progresso que $v_{k}$ faz em direção a satisfazer as restrições de igualdade linearizadas, porém ao mesmo tempo em que minimiza a função objetivo.

Diferentemente do subproblema vertical, o conjunto de restrições do subproblema horizontal é composto pela restrição de região de confiança e pelas restrições de igualdade. No entanto, é possível reformular (7) sem restriçooes de igualdade de forma que (5) e (7) podem ser resolvidos pelo mesmo algoritmo. Para esse fim, Byrd e Omojokun propõem calcular o passo $d$ por um passo complementar a $v_{k}$. Tal passo é ortogonal a $v_{k}$ e pertence ao espaço nulo de $\nabla g\left(x_{k}\right)$.

Seja $Z_{k}$ uma matriz $n \times(n-m)$ que gera o espaço nulo de $\nabla g\left(x_{k}\right)$, ou seja, $\nabla g\left(x_{k}\right)^{T} Z_{k}=0$. Então, o passo total $d$ pode ser decomposto como:

$$
d=v_{k}+Z_{k} u
$$

em que $u \in \mathbb{R}^{n-m}$ é agora o vetor a ser determinado. Substituindo (8) em (7), observando que $v_{k}$ e $Z_{k} u$ são ortogonais, e ignorando os termos constantes, o subproblema horizontal (7) pode ser reescrito sem restrições de igualdade, como

$$
\begin{aligned}
\min & \nabla \bar{f}_{k}\left(x_{k}\right)^{T} Z_{k} u+\frac{1}{2} u^{T} Z_{k}^{T} H_{k} Z_{k} u \\
\text { sujeito a } & \left\|Z_{k} u\right\| \leq \sqrt{\Delta_{k}^{2}-\left\|v_{k}\right\|^{2}}
\end{aligned}
$$

em que $\nabla \bar{f}_{k}\left(x_{k}\right)=\nabla f_{k}\left(x_{k}\right)+H_{k} v_{k}$. O procedimento para resolver (2) encontra-se agora delineado. Primeiro, resolve-se (5) para o passo vertical $v_{k}$, a seguir, resolve-se (9) para o passo horizontal $Z_{k} u_{k}$, depois, utilizando (8), calcula-se o passo completo $d_{k}$ que aproximadamente resolve (2), para finalmente obter

$$
x_{k+1}=x_{k}+d_{k}
$$

desde que $x_{k+1}$ proporcione uma redução na função mérito (a ser definida); caso contrário, a região de confiança é reduzida e um novo ponto tentativa é calculado.

\section{MÉTODO DE PONTOS-INTERIORES}

Para a apresentação do algoritmo de pontos-interiores para a solução dos subproblemas vertical e horizontal, define-se uma notação e forma padrão comum aos dois subproblemas, como segue:

$$
\begin{aligned}
\min & \phi(w)=b_{k}^{T} w+\frac{1}{2} w^{T} A_{k} w \\
\text { sujeito a } & \underline{w} \leq w \leq \bar{w}
\end{aligned}
$$

em que o índice $k$ significa um subproblema na iteração $k$. Para modelar (5), define-se $w=v$,

$$
\begin{aligned}
b_{k} & =\nabla g\left(x_{k}\right) g\left(x_{k}\right) \\
A_{k} & =\nabla g\left(x_{k}\right) \nabla g\left(x_{k}\right)^{T} \\
C_{k} & =I \\
\underline{w} & =\max \left\{\delta_{\underline{x}},-\xi \Delta_{k}\right\} \\
\bar{w} & =\min \left\{\delta_{\bar{x}}, \xi \Delta_{k}\right\} .
\end{aligned}
$$

Para modelar (9), define-se $w=u$,

$$
\begin{aligned}
b_{k} & =Z_{k}^{T} \nabla \bar{f}_{k} \\
A_{k} & =Z_{k}^{T} H_{k} Z_{k} \\
C_{k} & =Z_{k} \\
\underline{w} & =\max \left\{\delta_{\underline{x}},-\Delta_{k}\right\}-v_{k} \\
\bar{w} & =\min \left\{\delta_{\bar{x}}, \Delta_{k}\right\}-v_{k} .
\end{aligned}
$$

O método primal-dual de pontos-interiores para resolver o problema (11) opera sobre um problema modificado através da adição de vetores de folgas $s \geq 0$ e $z \geq 0$, como segue:

$$
\begin{aligned}
\min & \phi(w) \\
\text { sujeito a } & \underline{w}+s-w=0, \quad s \geq 0, \\
& w+z-\bar{w}=0, \quad z \geq 0 .
\end{aligned}
$$

As condições de não-negatividade em (14) são incorporadas numa função de barreira logaritmica que é agregada a função objetivo, como

$$
\begin{array}{ll}
\min \quad \phi(w)-\mu_{k} \sum_{i=1}^{p}\left(\ln s_{i}+\ln z_{i}\right) \\
\text { sujeito a } \quad \underline{w}+s-w=0, \quad s>0, \\
w+z-\bar{w}=0, \quad z>0,
\end{array}
$$

em que $\mu_{k}>0$ é o parâmetro de barreira que é monotonicamente reduzido para zero quando as iterações avançam. As condições de positividade estrita $s>0$ e $z>0$ devem ser impostas para que os termos logaritmicos sejam definidos. Essas condições são tratadas implicitamente, através do controle do tamanho do passo. 
As condições necessárias de otimalidade para (15) podem ser derivadas a partir de uma função de Lagrange associada ao problema,

$$
\begin{aligned}
L\left(y ; \mu_{k}\right)=\phi(w)-\mu_{k} \sum_{i=1}^{p}\left(\ln s_{i}+\ln z_{i}\right)+ \\
\pi^{T}(\underline{w}+s-w)+v^{T}(w+z-\bar{w})
\end{aligned}
$$

em que $\pi \in \mathbb{R}_{+}^{p}$ e $v \in \mathbb{R}_{+}^{p}$ são os multiplicadores de Lagrange e $y=(s, z, \pi, v, w)$. Os passos principais do algoritmo primal-dual de pontos-interiores são:

S1 Faça $k=0$, escolha $\mu_{0}>0$ e um ponto inicial $y_{0}$ que satisfaça as condições de estrita positividade $\left(s_{0}, z_{0}, \pi_{0}, v_{0}\right)>0$.

S2 Obtenha o sistema de Newton no ponto $y_{k}$,

$$
\nabla_{y y}^{2} L\left(y_{k} ; \mu_{k}\right) \Delta y=-\nabla_{y} L\left(y_{k} ; \mu_{k}\right),
$$

e resolva para a direção de Newton $\Delta y$.

S3 Calcule o comprimento de passo $\alpha_{k}$ na direção $\Delta y$ e obtenha uma nova estimativa da solução como $y_{k+1}=y_{k}+\alpha_{k} \Delta y$.

$S_{4}$ Se $y_{k+1}$ satisfaz o critério de convergência, então FIM. Caso contrário, faça $k \leftarrow k+1$, reduza $\mu_{k}$, e retorne para o passo 2 .

No passo S2, o sistema de Newton é da forma

$$
\left[\begin{array}{ccccc}
\Pi & 0 & S & 0 & 0 \\
0 & \Upsilon & 0 & Z & 0 \\
I & 0 & 0 & 0 & -I \\
0 & I & 0 & 0 & I \\
0 & 0 & -I & I & \nabla^{2} \phi(w)
\end{array}\right]\left(\begin{array}{c}
\Delta s \\
\Delta z \\
\Delta \pi \\
\Delta v \\
\Delta w
\end{array}\right)=-\left(\begin{array}{l}
S \pi-\mu_{k} e \\
Z v-\mu_{k} e \\
w+s-w \\
w+z-\bar{w} \\
\nabla \phi(w)-\pi+v
\end{array}\right)
$$

em que $\Pi=\operatorname{diag}\left(\pi_{1}, \ldots, \pi_{p}\right), \Upsilon=\operatorname{diag}\left(v_{1}, \ldots, v_{p}\right)$ e $\nabla^{2} \phi(w)$ é a Hessiana de $\phi(w)$.

No passo $S 3$, os comprimentos de passo máximos das variáveis primais e duais na direção $\Delta y$ são dados por

$$
\begin{aligned}
& \alpha_{k}^{P}=\min \left\{1, \gamma \min _{\Delta_{i}<0}\left\{\frac{-s_{i}^{k}}{\Delta s_{i}}, \frac{-z_{i}^{k}}{\Delta z_{i}}\right\}\right\}, \\
& \alpha_{k}^{D}=\min \left\{1, \gamma \min _{\Delta_{i}<0}\left\{\frac{-\pi_{i}^{k}}{\Delta \pi_{i}}, \frac{-v_{i}^{k}}{\Delta v_{i}}\right\}\right\} .
\end{aligned}
$$

O escalar $\gamma \in(0,1)$ é um fator de segurança para assegurar que o próximo ponto satisfará as condições de estrita positividade; um valor típico é $\gamma=0.99995$.

No passo $S_{4}$, o parâmetro de barreira $\mu_{k}$ é reduzido em função do decréscimo do resíduo de complementaridade,

$$
\mu_{k+1}=\sigma \frac{s_{k+1}^{T} \pi_{k+1}+z_{k+1}^{T} v_{k+1}}{2 p}
$$

em que $\sigma=0.2$ é o parâmetro de centralização. As iterações de pontos-interiores terminam assim que

$$
\begin{aligned}
\max _{i}\left\{\underline{w}_{i}-w_{i}^{k}\right\}, \max _{i}\left\{w_{i}^{k}-\bar{w}_{i}\right\} & \leq \epsilon_{1}, \\
\frac{\left\|\nabla \phi\left(w_{k}\right)-\pi_{k}+v_{k}\right\|_{\infty}}{1+\left\|w_{k}\right\|_{2}+\left\|\pi_{k}\right\|_{2}+\left\|v_{k}\right\|_{2}} & \leq \epsilon_{1}, \\
\frac{s_{k}^{T} \pi_{k}+z_{k}^{T} v_{k}}{1+\left\|w_{k}\right\|_{2}} & \leq \epsilon_{2} .
\end{aligned}
$$

Tolerâncias típicas são $\epsilon_{1}=10^{-4}$ e $\epsilon_{2}=10^{-2} \epsilon_{1}$.

\section{ASPECTOS DA IMPLEMENTAÇÃO COMPUTACIONAL}

Nesta seção são discutidos os principais aspectos da implementação computacional do algoritmo de região de confiança.

\subsection{Os Multiplicadores de Lagrange}

Os multiplicadores de Lagrange associados com o FPO não-linear (1) não são explicitamente calculados pelo método de região de confiança. Assim, para calcular $H_{k}$ em cada iteração de região de confiança, dada por

$$
H_{k}=\nabla^{2} f\left(x_{k}\right)+\sum_{i=1}^{m} \lambda_{i}^{k} \nabla^{2} g_{i}\left(x_{k}\right),
$$

pode-se utilizar uma estimativa de mínimos quadrados para os multiplicadores de Lagrange, dada por

$$
\left(\nabla g\left(x_{k}\right)^{T} \nabla g\left(x_{k}\right)\right) \lambda_{k}=\nabla g\left(x_{k}\right)^{T} \nabla f\left(x_{k}\right) .
$$

Sob a hipótese de qualificação das restrições (condição de regularidade), a matriz de gradientes $\nabla g\left(x_{k}\right)$ é de posto completo e o sistema definido positivo (21) pode ser resolvido de forma eficiente por fatorização Cholesky ou um método de gradiente conjugado. A avaliação de $H_{k}$ pode ser implementada de forma bastante eficiente como descrito em (Torres e Carvalho Jr., 2006).

O sistema linear (21) é derivado da equação KKT dual do problema de FPO sem restrições de limites. Todavia, devido a restrição (1c), a equação KKT dual é da forma

$$
\nabla f(x)+\nabla g(x) \lambda-\widetilde{\pi}+\widetilde{v}=0 .
$$

Como há condições de sinais sobre os multiplicadores de Lagrange $\tilde{\pi}$ e $\widetilde{v}$ associados com as restrições de limites mínimos e máximos, respectivamente, um procedimento especial para o cálculo de $\lambda_{k}$ é descrito em (Plantenga, 1994).

Neste artigo, tira-se proveito da solução do subproblema horizontal pelo método de pontos-interiores para estimar de forma simples e computacionalmente barata os 
multiplicadores de Lagrange. Simplesmente define-se $\lambda_{k}=\tau_{*}$, em que $\tau_{*}$ é o multiplicador de Lagrange da restrição de igualdade linear do subproblema horizontal na iteração $k$. Esta estimativa é precisa e prontamente disponível após a solução do subproblema horizontal (7) em cada iteração de região de confiança.

\subsection{Função Mérito}

Uma vez resolvido (7) para o passo $d_{k}$, uma função mérito é utilizada para decidir se esse passo proporciona um decréscimo suficiente ou não na função objetivo não-linear $f(x)$. Baseado em (Omojokun, 1989; Lalee et al., 1998), a seguinte função mérito é utilizada

$$
\psi(x, \eta)=f(x)+\eta\|g(x)\|_{2}
$$

em que $\eta>0$ é um parâmetro de penalidade que pondera a satisfação das restrições relativo a minimização da função objetivo. Um procedimento para escolha e atualização de $\eta$ é descrito em (Lalee et al., 1998).

Basicamente, dado um passo $d_{k}, \eta$ deve ser escolhido grande o suficiente para que $d_{k}$ resulte numa redução do modelo da função mérito em $x_{k}$,

$$
\begin{gathered}
\tilde{\psi}(d, \eta)=f\left(x_{k}\right)+\nabla f\left(x_{k}\right)^{T} d+\frac{1}{2} d^{T} H_{k} d+ \\
\eta\left\|g\left(x_{k}\right)+\nabla g\left(x_{k}\right)^{T} d\right\|_{2} .
\end{gathered}
$$

O modelo da função mérito (24) é utilizado para calcular a redução prevista na função mérito (23), definida como sendo a variação no modelo $\tilde{\psi}(d, \eta)$ devida a um passo $d$, ou seja,

$$
\begin{aligned}
\operatorname{rp}\left(d_{k}\right) & =\tilde{\psi}(0, \eta)-\tilde{\psi}\left(d_{k}, \eta\right) \\
& =\psi\left(x_{k}, \eta\right)-\tilde{\psi}\left(d_{k}, \eta\right) .
\end{aligned}
$$

$\operatorname{rp}\left(d_{k}\right)$ é utilizado como uma medida para aceitar o passo $d_{k}$ e para atualizar a região de confiança. Compara-se $\operatorname{rp}\left(d_{k}\right)$ com a redução real na função mérito $\psi(x, \eta)$, dada por

$$
\operatorname{rr}\left(d_{k}\right)=\psi\left(x_{k}, \eta\right)-\psi\left(x_{k}+d_{k}, \eta\right) .
$$

Uma taxa de redução $\rho_{k}$ é então definida como

$$
\rho_{k}=\frac{\operatorname{rr}\left(d_{k}\right)}{\operatorname{rp}\left(d_{k}\right)}=\frac{\psi\left(x_{k}, \eta\right)-\psi\left(x_{k}+d_{k}, \eta\right)}{\psi\left(x_{k}, \eta\right)-\tilde{\psi}\left(d_{k}, \eta\right)} .
$$

Como explicado em (Nocedal e Wright, 1999), se $\rho_{k}$ é próximo de 1 então há uma boa concordância entre o modelo $\tilde{\psi}_{k}$ e a função mérito $\psi$ ao longo do passo $d$ considerado, de forma que é seguro expandir a região de confiança na próxima iteração. Se $\rho_{k}$ é positivo mas não próximo de 1, então o tamanho da região é mantido. Mas se ele é próximo de zero ou negativo a região de confiança é reduzida.
A condição base para a escolha e atualização da penalidade $\eta$ é que ela deve ser grande o suficiente para $d_{k}$ proporcionar uma redução em $\tilde{\psi}(d, \eta)$. Primeiro, um valor tentativa $\eta^{+}$é obtido como:

$\eta^{+}=\max \left\{\eta_{k}, 0.1+\frac{\nabla f\left(x_{k}\right)^{T} d_{k}+\frac{1}{2} d_{k}^{T} H_{k} d_{k}}{\left\|g\left(x_{k}\right)\right\|-\left\|g\left(x_{k}\right)+\nabla g\left(x_{k}\right)^{T} v_{k}\right\|}\right\}$

em que $\eta_{k}$ é penalidade utilizada na iteração anterior. Em seguida, o valor tentativa é melhorado conforme o seguinte algoritmo proposto em (Lalee et al., 1998):

$S 1 \quad$ Se $\left\|g\left(x_{k}\right)\right\|>\frac{1}{5}\left\|g\left(x_{k-l}\right)\right\|, \eta^{+}>\eta_{k}, \eta^{+}<5 \eta_{k}$ $\eta_{k}>\eta_{k-l}$, e um dos dois últimos passos falhou,

$$
\eta^{+}=\min \left\{5 \eta_{k}, \eta^{+}+25\left(\eta^{+}-\eta_{k-l}\right)\right\}
$$

em que $k-l$ é o índice do último passo de sucesso.

S2 Se $\eta^{+}=\eta_{k},\left\|v_{k}\right\|<\frac{\xi \Delta_{k}}{10}$ e $\left\|g\left(x_{k}\right)\right\|_{\infty}<10^{4} \epsilon$,

$$
\eta^{+}=\max \left\{\eta_{0}, \bar{\eta},\left\|\lambda_{k}\right\|\right\}
$$

em que $\bar{\eta}$ é o segundo termo entre chaves em (28).

As atualizações do raio da região de confiança, $\Delta_{k}$, e do parâmetro de penalidade, $\eta_{k}$, são como segue:

$S 1$ Calcule $\eta^{+}$conforme o algoritmo anterior, $\operatorname{rr}\left(d_{k}\right)$ por $(26)$, e $\operatorname{rp}\left(d_{k}\right)$ por $(25)$.

S2 Se $\frac{\operatorname{rr}\left(d_{k}\right)}{\operatorname{rp}\left(d_{k}\right)} \geq \bar{\rho}$, então faça $x_{k+1}=x_{k}+d_{k}, \eta_{k+1}=$ $\eta^{+}, \Delta_{k+1} \geq \Delta_{k}$, e retorne.

S3 Se $\left\|v_{k}\right\| \leq 0.8 \xi \Delta_{k}$ e $\left\|v_{k}\right\| \leq 0.1\left\|Z_{k} u_{k}\right\|$, então obtenha a correção de segunda ordem

$d_{\text {so }}=d_{k}-\nabla g\left(x_{k}\right)\left(\nabla g\left(x_{k}\right)^{T} \nabla g\left(x_{k}\right)\right)^{-1} g\left(x_{k}+d_{k}\right)$

calcule $\operatorname{rr}\left(d_{\mathrm{so}}\right)$ e vá para o passo $S_{4}$. Senão, faça $x_{k+1}=x_{k}, \eta_{k+1}=\eta_{k}, \Delta_{k+1} \leq \gamma\left\|d_{k}\right\|$ e retorne.

$S_{4}$ Se $\frac{\operatorname{rr}\left(d_{\mathrm{so}}\right)}{\operatorname{rp}\left(d_{k}\right)} \geq \bar{\rho}$, então faça $x_{k+1}=x_{k}+d_{\mathrm{so}}$, $\eta_{k+1}=\eta^{+}, \Delta_{k+1} \geq \Delta_{k}$, e retorne. Senão, faça $x_{k+1}=x_{k}, \eta_{k+1}=\eta_{k}, \Delta_{k+1} \leq \gamma\left\|d_{k}\right\|$ e retorne.

A atualização do raio da região de confiança descrito no algoritmo simplesmente como $\Delta_{k+1} \geq \Delta_{k}$ ou $\Delta_{k+1} \leq$ $\gamma\left\|d_{k}\right\|$, com $\gamma \in(0,1)$, é como segue. Baseado em (Plantenga, 1994), quando um passo $d_{k}$ é aceito o raio 
da região de confiança é aumentado como:

$$
\Delta_{k+1}= \begin{cases}\max \left\{2\left\|d_{k}\right\|, \Delta_{k}\right\} & \text { se } \frac{\operatorname{rr}\left(d_{k}\right)}{\operatorname{rp}\left(d_{k}\right)} \geq 0.9 \\ \max \left\{1.2\left\|d_{k}\right\|, \Delta_{k}\right\} & \text { se } 0.3 \leq \frac{\operatorname{rr}\left(d_{k}\right)}{\operatorname{rp}\left(d_{k}\right)}<0.9 \\ \Delta_{k} & \text { caso contrário }\end{cases}
$$

Quando o passo $d_{k}$ é rejeitado, $\Delta_{k+1}$ é reduzido para uma fração $\gamma \in[0.1,0.5]$ desse passo, com $\gamma$ dado por

$$
\gamma= \begin{cases}\frac{1-\bar{\rho}}{1-\frac{\operatorname{rr}\left(d_{k}\right)}{\operatorname{rp}\left(d_{k}\right)}} & \text { se }\left\|d_{k}\right\|=\Delta_{k} \\ \frac{\dot{p}(0)}{2\left(\dot{p}(0)-\frac{\operatorname{rr}\left(d_{k}\right)}{\operatorname{rp}\left(d_{k}\right)}\right)} & \text { caso contrário }\end{cases}
$$

em que $\dot{p}(0)=-\nabla f\left(x_{k}\right)^{T} d_{k}+\eta_{k+1}\left\|\nabla g\left(x_{k}\right)^{T} d_{k}\right\|$. Em ambos os casos o valor de $\gamma$ é restrito ao intervalo $[0.1,0.5]$.

Os passos básicos da técnica de Byrd-Omojokun são como segue:

S1 Faça $k=0$, escolha $x_{0}$ dentro de limites, escolha um tamanho máximo da região de confiança $\bar{\Delta}>$ $0, \Delta_{0} \in(0, \bar{\Delta}), \epsilon \in\left(0, \frac{1}{2}\right]$ e $\bar{\rho} \in\left(0, \frac{1}{2}\right]$.

S2 Se $\left\|\nabla f\left(x_{k}\right)+\nabla g\left(x_{k}\right)^{T} \lambda_{k}\right\|<\epsilon$ e $\left\|g\left(x_{k}\right)\right\|_{\infty}<\epsilon$, então pare.

S3 Resolva o subproblema vertical (5) para o passo $v_{k}$.

S4 Resolva o subproblema horizontal (7) para o passo $u_{k}$.

S5 Calcule a solução do subproblema (2) como $d_{k}=$ $v_{k}+Z_{k} u_{k}$.

S6 Calcule $\operatorname{rp}\left(d_{k}\right), \operatorname{rr}\left(d_{k}\right)$ e $\eta^{+}$por (25) a (28).

$S 7$ Calcule $x_{k+1}, \eta_{k+1}$ e $\Delta_{k+1}$. Faça $k=k+1$ e retorne para o passo 52 .

\section{RESULTADOS NUMÉRICOS}

O algoritmo de região de confiança com resolução dos subproblemas pelo método primal-dual de pontosinteriores para programação quadrática é aplicado nos sistemas testes do IEEE com 30, 57, 118 e 300 barras. O algoritmo de região de confiança com pontosinteriores (RCPI) é comparado a dois algoritmos de pontos-interiores para a solução direta do FPO nãolinear: o método primal-dual simples (PDPI) e a sua variante tipo preditor-corretor (PDPC). O problema de FPO resolvido é o de minimização de perdas ativas.

O conjunto de restrições de igualdade (1b) inclui as equações de balanço de potência ativa e potência reativa em todas as barras. As restrições de desigualdade (1c) incluem limites mínimos e máximos sobre os módulos das tensões, os tapes dos transformadores com LTC, a potência reativa dos geradores, e as compensações de reativo em paralelo. As implementações dos algoritmos foram totalmente desenvolvidas na linguagem MATLAB, e por essa razão análise crítica e comparações de tempo de processamento não são apresentadas.

Os algoritmos de pontos-interiores PDPI e PDPC para solução direta não-linear iniciam as variáveis de folga e multiplicadores de Lagrange conforme sugerido em (Torres e Quintana, 1998). Para fins de comparação dos algoritmos, foram implementadas quatro opções de escolha do ponto $x_{0}$ : (i) uma solução inicial de fluxo de potência, (ii) uma inicialização "flat" (ou seja, $V_{i}^{0}=1$ e $\theta_{i}^{0}=0$ ), (iii) o ponto médio entre os limites (por exemplo, $\left.V_{i}^{0}=\left(V_{i}^{\min }+V_{i}^{\max }\right) / 2\right)$, ou (iv) pontos aleatórios dentro dos limites. A função MATLAB rand $(n, 1)$ gera um vetor de $n$ elementos aleatórios uniformemente distribuídos no intervalo $[0,1]$, de maneira que vetores de números aleatórios uniformemente distribuídos no intervalo $[\underline{x}, \bar{x}]$ podem ser gerados por

$\mathrm{x}=\mathrm{x}_{-} \min +\operatorname{rand}(\mathrm{n}, 1) . *\left(\mathrm{x}_{-} \max -\mathrm{x}_{-} \min \right)$

As inicializações (ii), (iii) e (iv) satisfazem as restrições de limites mas violam as restrições de igualdade. A inicialização (i) satisfaz as restrições de igualdades mas provavelmente viola limites. No entanto, observa-se de (4c) que um ponto inicial não pode violar um limite por um montante maior do que o raio inicial da região de confiança, $\Delta_{0}$. Caso contrário, o limite inferior em (4c) será maior do que o limite superior e o subproblema de região de confiança é mau-posto. Em resumo, $x_{0}$ não precisa satisfazer as restrições de igualdade mas deve estar dentro do intervalo $\left(\underline{x}-\Delta_{0}, \bar{x}+\Delta_{0}\right)$. As regras (ii), (iii) e (iv) naturalmente atendem este requisito.

A inicialização dos algoritmos de pontos-interiores para PQ na resolução dos subproblemas vertical e horizontal é bem mais simples, com $v_{0}=0$ e $d_{0}=v_{*}$, em que $v_{*}$ é a solução ótima do subproblema vertical resolvido antes do subproblema horizontal. Os parâmetros utilizados pelos algoritmos de pontos-interiores são: $\mu_{0}=0,01$, $\gamma=0,99995, \sigma=0,2$ e $\epsilon=10^{-4}$. Os parâmetros utilizados pelo algoritmo de região de confiança são: $\Delta_{0}=1$, $\bar{\Delta}=5, \eta_{0}=2$ e $\bar{\rho}=0,1$. O complemento ortogonal $Z_{k}$ é calculado através do comando MATLAB null ( ) tendo a matriz $\nabla g\left(x_{k}\right)$ como argumento.

A Tabela 1 exibe, para cada sistema, o número total de barras $|\mathcal{N}|$, o número de barras de geração $|\mathcal{G}|$, o número de barras de carga com controle de reativo em paralelo $|\mathcal{E}|$, o número total de circuitos (linhas e transformadores) $|\mathcal{B}|$, e o número de transformadores com LTC $|\mathcal{T}|$. A tabela também apresenta o número total de variáveis 
primais, $n$, o número de restrições de igualdades, $m$, e número de restrições com limites duplos, $p$. A Tabela 2 exibe a carga ativa e reativa total, e as perdas ativas para a condição base de operação, ou seja, antes da aplicação de qualquer procedimento de otimização.

Tabela 1: Dimensões dos sistemas testes e dos problemas de FPO correspondentes

\begin{tabular}{|l|rrrrr|rrr|}
\hline Sistema & $|\mathcal{N}|$ & $|\mathcal{G}|$ & $|\mathcal{E}|$ & $|\mathcal{B}|$ & $|\mathcal{T}|$ & $n$ & $m$ & $p$ \\
\hline IEEE 30 & 30 & 6 & 5 & 41 & 4 & 75 & 60 & 46 \\
IEEE 57 & 57 & 7 & 5 & 80 & 17 & 143 & 114 & 87 \\
IEEE 118 & 118 & 54 & 12 & 186 & 9 & 311 & 236 & 194 \\
IEEE 300 & 300 & 69 & 23 & 411 & 35 & 727 & 600 & 428 \\
\hline
\end{tabular}

Tabela 2: Carga total ativa e reativa, e perdas ativas.

\begin{tabular}{|l|rrr|}
\hline Sistemas & $P(\mathrm{MW})$ & $Q(\mathrm{MVAr})$ & $P_{L}(\mathrm{MW})$ \\
\hline IEEE 30 & 283,40 & 126,20 & 17,63 \\
IEEE 57 & $1.250,80$ & 336,40 & 27,86 \\
IEEE 118 & $3.668,00$ & $1.438,00$ & 132,48 \\
IEEE 300 & $23.246,87$ & $7.787,97$ & 408,32 \\
\hline
\end{tabular}

Tabela 3: Número de iterações e perda ativa final.

\begin{tabular}{|l|ccc|rr|}
\hline \multirow{2}{*}{ Sistemas } & \multicolumn{2}{|c|}{ Número de iterações } & \multicolumn{2}{c|}{ Perda ativa final } \\
& PDPI & PDPC & RCPI & (MW) & Red.(\%) \\
\hline IEEE 30 & 10 & 7 & 2 & 17.79 & -0.91 \\
IEEE 57 & 11 & 8 & 3 & 24.34 & 12.63 \\
IEEE 118 & 14 & 9 & 3 & 118.77 & 10.36 \\
IEEE 300 & 15 & 9 & 4 & 378.37 & 7.33 \\
\hline
\end{tabular}

Tabela 4: Número de iterações por RCPI usando inicialização (i)

\begin{tabular}{|l|c|rrr|rr|}
\hline \multirow{2}{*}{ Sistema } & \multirow{2}{*}{$\Delta_{0}$} & \multicolumn{3}{|c|}{ Iterações } & \multicolumn{2}{c|}{ Média Iterações } \\
& & RCPI & PI-V & PI-H & mPI-V & mPI-H \\
\hline IEEE 30 & 1 & 2 & 12 & 12 & 6.0 & 6.0 \\
IEEE 57 & 1 & 3 & 18 & 19 & 6.0 & 6.3 \\
IEEE 118 & 1 & 3 & 19 & 22 & 6.3 & 7.3 \\
IEEE 300 & 1 & 4 & 31 & 38 & 7.7 & 9.5 \\
\hline
\end{tabular}

Para a inicialização (i) (fluxo de potência), a Tabela 3 mostra o número de iterações para convergência pelos algoritmos PDPI e PDPC na solução direta do FPO não-linear, o número de iterações externas do algoritmo RCPI, e as perdas ativas após a otimização, em MW e percentagem de redução. Os pontos de operação ótimos obtidos pelos três algoritmos (PDPI, PDPC e RCPI) são exatamente os mesmos. Todos os algoritmos tiveram sucesso na otimização de todos os sistemas. Como já relatado na literatura, o algoritmo PDPC apresenta desempenho melhor do que o algoritmo PDPI. Observa-se que o número de iterações de região de confiança é pequeno (3 a 4 iterações por sistema). Todavia, devido ao custo (tempo de processamento) de uma iteração RCPI ser aproximadamente 1,5 vezes o custo de uma solução
Tabela 5: Número de iterações, inicializações (ii) e (iii)

\begin{tabular}{|l|rrr|rrr|}
\hline \multirow{2}{*}{ Sistema } & \multicolumn{3}{|c|}{ Iterações - (ii) } & \multicolumn{3}{|c|}{ Iterações - (iii) } \\
& PDPI & PCPI & RCPI & PDPI & PCPI & RCPI \\
\hline IEEE 30 & 11 & 9 & 3 & 11 & 9 & 3 \\
IEEE 57 & 13 & 12 & 5 & 12 & 10 & 5 \\
IEEE 118 & 14 & 11 & 4 & 13 & 9 & 4 \\
IEEE 300 & 19 & 12 & 5 & 18 & 12 & 5 \\
\hline
\end{tabular}

Tabela 6: Número de iterações por RCPI usando inicialização (ii)

\begin{tabular}{|l|c|rrr|rr|}
\hline \multirow{2}{*}{ Sistema } & \multirow{2}{*}{$\Delta_{0}$} & \multicolumn{2}{|c|}{ Iterações - (ii) } & \multicolumn{2}{c|}{ Média Iterações } \\
& & RCPI & PI-V & PI-H & mPI-V & mPI-H \\
\hline IEEE 30 & 1 & 3 & 18 & 18 & 6.0 & 6.0 \\
IEEE 57 & 1 & 5 & 30 & 30 & 6.0 & 6.0 \\
IEEE 118 & 1 & 4 & 31 & 31 & 7.7 & 7.7 \\
IEEE 300 & 1 & 5 & 45 & 44 & 9.0 & 8.8 \\
\hline
\end{tabular}

Tabela 7: Número de iterações por RCPI usando inicialização (iii)

\begin{tabular}{|l|c|rrr|rr|}
\hline \multirow{2}{*}{ Sistema } & \multirow{2}{*}{$\Delta_{0}$} & \multicolumn{3}{|c|}{ Iterações - (iii) } & \multicolumn{2}{c|}{ Média Iterações } \\
& & RCPI & PI-V & PI-H & mPI-V & mPI-H \\
\hline IEEE 30 & 1 & 3 & 18 & 18 & 6.0 & 6.0 \\
IEEE 57 & 1 & 5 & 33 & 31 & 6.6 & 6.2 \\
IEEE 118 & 1 & 4 & 31 & 30 & 7.7 & 7.5 \\
IEEE 300 & 1 & 5 & 45 & 44 & 9.0 & 8.8 \\
\hline
\end{tabular}

PDPI, o algoritmo RCPI é aproximadamente 6 vezes mais lento do que o algoritmo PDPI. A Tabela 4 detalha a solução pelo algoritmo RCPI. As Tabelas 5, 6 e 7 detalham o desempenho do algoritmo RCPI quando o ponto inicial é escolhido pelas regras (ii) e (iii). Claramente o desempenho do algoritmo RCPI é similar nas três regras de escolha do ponto inicial.

A Tabela 8 detalha o processo de convergência para o sistema teste IEEE 300 barras. A coluna $f\left(x_{k}\right)$ é a função objetivo não-linear em (1); as colunas PI-V e PI-H são os números de iterações de pontos-interiores para resolver os subproblemas vertical e horizontal, respectivamente, em cada iteração externa; Primal e Dual são os resíduos de inviabilidade primal e dual, respectivamente; $r$ r/rp é a taxa de reduão $\operatorname{rr}\left(d_{k}\right) / \operatorname{rp}\left(d_{k}\right) ; \Delta_{k}$ é o tamanho do região de confiança; e $\eta_{k}$ é o parâmetro de penalidade na função mérito. A função objetivo pode aumentar em algumas iterações, uma vez que a função mérito pondera entre reduzir a função objetivo e satisfazer as restrições, e pontos inviáveis durante as iterações podem proporcionar valores da função objetivo menores. O tamanho da região de confiança aumenta durante as iterações. A inviabilidade dual nas iterações externas (mostrada na Tabela 8) é apenas uma aproximação, uma vez que os multiplicadores de Lagrange das restrições de limites do 
problema não-linear (1) não são calculados pelo método de região de confiança; os valores exibidos levam em conta apenas as restrições de igualdades e utiliza uma estimativa dos multiplicadores de Lagrange.

O número de iterações de pontos-interiores para resolver os subproblemas vertical e horizontal varia muito pouco. O maior número de iterações de pontos-interiores geralmente ocorre na solução do subproblema vertical nas primeiras iterações de região de confiança, e quando o ponto $x_{0}$ é distante da solução ótima. As Tabelas $9 \mathrm{e}$ 10 demonstram o processo de convergência do algoritmo de pontos-interiores aplicado aos subproblemas vertical e horizontal na primeira iteração de região de confiança na otimização do sistema IEEE 300 barras. A coluna $k$ refere-se aos comprimentos de passos (comum) primal e dual; as colunas Primal e Dual são as inviabilidades primal e dual, respectivamente; e a coluna Objetivo exibe os valores da função objetivo, incluindo os termos constantes que foram desprezados em (6) e (7).

Para avaliar a robustez do algoritmo RCPI, dois conjuntos de simulações são executados. Um diz respeito a escolha do ponto inicial, onde um conjunto de 50 pontos iniciais aleatórios é gerado para cada sistema teste, e o outro diz respeito a forma do conjunto viável. Para o último caso, o carregamento do sistema é aumentado para estressar o sistema, de forma que a solução do FPO torne-se mais difícil de obter com os controles e limites operacionais especificados. Para gerar casos de difícil solução com a garantia de serem casos viáveis, o ponto de máximo carregamento do sistema que ainda satisfaz os limites operacionais especificados foi calculado resolvendo-se outro problema de FPO em que o parâmetro de carregamento é maximizado. A carga do sistema é então aumentada em $90 \%$ do máximo carregamento. As perdas ativas antes da otimização são 27,49 MW, 62,51 MW, 152,49 MW e 545,43 MW para os sistemas IEEE 30, 57, 118 e 300 barras, respectivamente. A Tabela 11 mostra os números de iterações para convergência e as perdas finais. O processo de convergência para o sistema IEEE 118 barras é mostrado na Tabela 12. Observa-se que o números de iterações RCPI não aumentou com a complexidade do problema de FPO.

A Tabela 13 resume o desempenho do algoritmo RCPI utilizando pontos iniciais aleatórios. Cinquenta pontos iniciais aleatórios foram gerados para cada sistema. A coluna Total refere-se ao número total de iterações de região de confiança no conjunto de 50 simulações; Med é o número médio de iterações de região de confiança; Max e Min são os números máximo e mínimo de iterações de região de confiança observados numa simulação; Max, Min e Med sob a coluna "Inviabilidades de $x_{0}$ " são
Tabela 8: Processo de convergência, IEEE 300 barras, inicialização (ii)

\begin{tabular}{|l|rr|cc|c|c|c|c|}
\hline \multirow{2}{*}{$k$} & \multicolumn{2}{|c|}{ Iterações } & \multicolumn{2}{|c|}{ Inviabilidades } & \multirow{2}{*}{$/ \mathrm{r}$} & $\Delta_{k}$ & $\eta_{k}$ & $f\left(x_{k}\right)$ \\
& PI-V & PI-H & Primal & Dual $(\approx)$ & $\mathrm{rr}$ & & & \\
\hline 0 & - & - & $8.64 \times 10^{-1}$ & $4.95 \times 10^{-4}$ & - & 1 & 2 & 8.20 \\
1 & 13 & 9 & $1.17 \times 10^{-1}$ & $5.43 \times 10^{-2}$ & 0.967 & 5 & 2 & 7.38 \\
2 & 14 & 9 & $2.81 \times 10^{-2}$ & $1.74 \times 10^{-2}$ & 0.964 & 5 & 2 & 4.38 \\
3 & 6 & 8 & $4.13 \times 10^{-3}$ & $2.17 \times 10^{-2}$ & 0.903 & 5 & 2 & 4.25 \\
4 & 6 & 9 & $7.92 \times 10^{-4}$ & $2.22 \times 10^{-2}$ & 0.706 & 5 & 2 & 4.26 \\
5 & 6 & 9 & $2.15 \times 10^{-5}$ & $2.22 \times 10^{-2}$ & 0.978 & 5 & 2 & 4.26 \\
\hline
\end{tabular}

Tabela 9: Processo de convergência, subproblema vertical, primeira iteração de RC, IEEE 300 barras

\begin{tabular}{|c|c|c|c|c|c|}
\hline \multirow[b]{2}{*}{$k_{i}$} & \multirow[b]{2}{*}{$\alpha_{k}$} & \multicolumn{3}{|c|}{ Inviabilidades } & \multirow{2}{*}{$\begin{array}{l}\text { Objetivo } \\
\text { em (5) }\end{array}$} \\
\hline & & Primal & Dual & Complem. & \\
\hline 0 & - & 0.0000 & $6.53 \times 10^{+3}$ & $1.45 \times 10^{+1}$ & $1.79 \times 10^{+3}$ \\
\hline 1 & 0.183 & 0.0000 & $5.21 \times 10^{+3}$ & $5.13 \times 10^{+0}$ & $1.20 \times 10^{+3}$ \\
\hline 2 & 0.558 & 0.0000 & $2.09 \times 10^{+3}$ & $1.15 \times 10^{+0}$ & $2.34 \times 10^{+2}$ \\
\hline 3 & 0.413 & $1.88 \times 10^{-4}$ & $1.00 \times 10^{+3}$ & $6.24 \times 10^{-1}$ & $8.08 \times 10^{+1}$ \\
\hline 10 & 0.746 & $3.84 \times 10^{-8}$ & $4.93 \times 10^{-2}$ & $6.32 \times 10^{-4}$ & $5.74 \times 10^{-1}$ \\
\hline 11 & 0.827 & $6.17 \times 10^{-9}$ & $8.51 \times 10^{-3}$ & $1.31 \times 10^{-4}$ & $5.73 \times 10^{-1}$ \\
\hline 12 & 0.880 & $0.00 \times 10^{+0}$ & $1.02 \times 10^{-3}$ & $2.20 \times 10^{-5}$ & $5.73 \times 10^{-1}$ \\
\hline 13 & 1.000 & $0.00 \times 10^{+0}$ & $9.04 \times 10^{-9}$ & $3.15 \times 10^{-6}$ & $5.73 \times 10^{-1}$ \\
\hline
\end{tabular}

Tabela 10: Processo de convergência, subproblema horizontal, primeira iteração de RC, IEEE 300 barras

\begin{tabular}{|r|c|ccc|c|}
\hline \multirow{2}{*}{$k_{i}$} & \multirow{2}{*}{$\alpha_{k}$} & \multicolumn{3}{|c|}{ Inviabilidades } & Objetivo \\
& & Primal & Dual & Complem. & em $(7)$ \\
\hline 0 & - & $0.00 \times 10^{+00}$ & $8.20 \times 10^{-2}$ & $1.34 \times 10^{+0}$ & $1.19 \times 10^{+1}$ \\
1 & 0.835 & $5.69 \times 10^{-03}$ & $1.47 \times 10^{-2}$ & $3.48 \times 10^{-1}$ & $1.18 \times 10^{+1}$ \\
2 & 0.602 & $2.42 \times 10^{-03}$ & $5.86 \times 10^{-3}$ & $1.59 \times 10^{-1}$ & $1.18 \times 10^{+1}$ \\
3 & 0.900 & $1.12 \times 10^{-14}$ & $5.86 \times 10^{-4}$ & $3.16 \times 10^{-2}$ & $1.18 \times 10^{+1}$ \\
\hline 6 & 0.707 & $5.25 \times 10^{-15}$ & $7.70 \times 10^{-6}$ & $8.48 \times 10^{-4}$ & $1.18 \times 10^{+1}$ \\
7 & 0.971 & $6.42 \times 10^{-15}$ & $2.27 \times 10^{-7}$ & $8.21 \times 10^{-5}$ & $1.18 \times 10^{+1}$ \\
8 & 0.838 & $1.06 \times 10^{-14}$ & $3.67 \times 10^{-8}$ & $2.23 \times 10^{-5}$ & $1.18 \times 10^{+1}$ \\
9 & 0.906 & $9.71 \times 10^{-11}$ & $3.47 \times 10^{-9}$ & $2.16 \times 10^{-6}$ & $1.18 \times 10^{+1}$ \\
\hline
\end{tabular}

os valores máximo, mínimo e médio do objetivo vertical na primeira iteração de região de confiança, respectivamente. Observa-se que o número de iterações de região de confiança muda muito pouco para o mesmo sistema, e também entre os diversos sistemas. Observa-se das últimas colunas Max e Min que os pontos iniciais aleatórios variam de média e elevada inviabilidade (o objetivo vertical é a norma do resíduo das restrições de igualdade).

\section{CONCLUSÕES}

O artigo apresentou de forma tutorial uma aplicação da técnica de região de confiança de Byrd e Omojokun na solução de problemas de FPO. A escolha da técnica de Byrd e Omojokun deve-se a sua eficiência no tratamento de inconsistência nas restrições dos subproblemas. O objetivo é o desenvolvimento de um algoritmo 
Tabela 11: Número de iterações e perdas finais: casos críticos

\begin{tabular}{|c|c|c|c|c|c|}
\hline \multirow{2}{*}{ Sistema } & \multicolumn{3}{|c|}{ Número de iterações } & \multicolumn{2}{|c|}{ Perda ativa final } \\
\hline & RCPI & PI-V & PI-H & $(\mathrm{MW})$ & Red.(\%) \\
\hline IEEE 30 & 3 & 18 & 20 & 27.74 & -0.91 \\
\hline IEEE 57 & 4 & 24 & 28 & 57.88 & 7.41 \\
\hline IEEE 118 & 4 & 31 & 30 & 137.97 & 9.52 \\
\hline IEEE 300 & 4 & 28 & 31 & 499.04 & 8.51 \\
\hline
\end{tabular}

Tabela 12: Processo de convergência, IEEE 118 barras, caso crítico

\begin{tabular}{|l|rr|cc|c|c|c|c|}
\hline \multirow{2}{*}{$k$} & \multicolumn{2}{|c|}{ Iterações } & \multicolumn{2}{|c|}{ Inviabilidades } & \multirow{2}{*}{ ar $/ \mathrm{pr}$} & $\Delta_{k}$ & $\eta_{k}$ & $f\left(x_{k}\right)$ \\
\hline & IP-V & IP-H & Primal & Dual $(\approx)$ & & & & \\
\hline 0 & - & - & $1.45 \times 10^{+0}$ & $7.64 \times 10^{-3}$ & - & 1 & 2 & 8.09 \\
1 & 13 & 7 & $2.45 \times 10^{-1}$ & $7.78 \times 10^{-2}$ & 0.905 & 5 & 2 & 8.89 \\
2 & 6 & 7 & $3.00 \times 10^{-2}$ & $2.59 \times 10^{-2}$ & 0.903 & 5 & 2 & 7.33 \\
3 & 6 & 8 & $6.11 \times 10^{-4}$ & $2.84 \times 10^{-2}$ & 0.982 & 5 & 2 & 7.52 \\
4 & 6 & 8 & $2.44 \times 10^{-6}$ & $2.86 \times 10^{-2}$ & 1.000 & 5 & 2 & 7.52 \\
\hline
\end{tabular}

Tabela 13: Cinquenta simulações por sistema, com o algoritmo RCPI usando pontos iniciais aleatórios

\begin{tabular}{|l|rrrr|rrr|}
\hline \multirow{2}{*}{ Sistema } & \multicolumn{3}{|c|}{ Número de Iterações } & \multicolumn{3}{|c|}{ Inviabilidade de $x_{0}$} \\
& Total & Med & Max & Min & Max & Min & Med \\
\hline IEEE 30 & 206 & 4.12 & 6 & 3 & 12.7 & 4.7 & 8.4 \\
IEEE 57 & 264 & 5.28 & 9 & 3 & 20.0 & 12.7 & 15.4 \\
IEEE 118 & 208 & 4.16 & 5 & 4 & 72.4 & 54.2 & 61.9 \\
IEEE 300 & 273 & 5.46 & 9 & 4 & 524.2 & 154.7 & 307.1 \\
\hline
\end{tabular}

de FPO globalmente convergente sem comprometimento do tempo de solução. Para a resolução dos subproblemas de região de confiança utilizou-se o método primaldual de pontos-interiores para $\mathrm{PQ}$, visando a fácil integração do algoritmo de região de confiança aos algoritmos de FPO baseados em métodos de pontos-interiores (Granville, 1994; Torres e Quintana, 1998). Os resultados obtidos até então demonstram a robustez da técnica de região de confiança na solução de problemas de FPO. Salienta-se que a ênfase da técnica de região de confiança é a robustez de convergência e não o tempo de processamento. No entanto, as pesquisas recentes com métodos de região de confiança têm tido como foco também a redução no esforço computacional, sem detrimento da robustez de convergência, visando aplicações em problemas de grande porte.

\section{AGRADECIMENTOS}

Os autores agradecem o apoio a pesquisa recebido das agências de fomento CNPq e CAPES.

\section{REFERÊNCIAS}

Almeida, K. C. (2006). Um estudo sobre casos críticos de solução do fluxo de potência ótimo, SBA Controle
E) Automação 17(1): 71-80.

Byrd, R. H. (1987). Robust trust region methods for constrained optimization, Proceedings of Third SIAM Conference on Optimization, Houston, TX.

Conn, A. R., Gould, N. I. M. e Toint, P. L. (2000). Trust-Region Methods, MPS-SIAM Series on Optimization, Philadelphia.

Costa, A. S., Salgado, R. e Haas, P. (2007). Globally convergent state estimation based on Givens rotations, Proc. of the 2007 IREP Symposium, Charleston SC, USA.

Gomes, F. A. M., Maciel, M. C. e Martínez, J. M. (1999). Nonlinear programming algorithms using trust regions and augmented lagrangians with nonmonotone penalty parameters, Mathematical Programming 84(1): 161-200.

Granville, S. (1994). Optimal reactive dispatch through interior point methods, IEEE Trans. on Power Systems 9(1): 136-146.

Lalee, M., Nocedal, J. e Plantenga, T. (1998). On the implementation of an algorithm for large-scale equality constrained optimization, SIAM J. on $O p$ timization 8(3): 682-706.

Moré, J. J. (1983). Iterative solution of linear systems, Mathematical Programming: The State of the Art, Springer Verlag, pp. 258-287.

Nocedal, J. e Wright, S. J. (1999). Numerical Optimization, Springer.

Omojokun, O. (1989). Trust Region Algorithms for Optimization with Nonlinear Equality and Inequality Constraints, PhD thesis, Dept. of Computer Science, University of Colorado, Boulder, Colorado.

Pajic, S. e Clements, K. A. (2003). Globally convergent state estimation via the trust region method, 2003 IEEE Bologna PowerTech Conference, Bologna, Italy.

Plantenga, T. D. (1994). Large-scale nonlinear constrained optimization using trust regions, $\mathrm{PhD}$ thesis, Northwestern University, Evanston, IL, USA.

Sorensen, D. C. (1982). Newton's method with a model trust region modification, SIAM J. on Numerical Analysis 19: 409-426.

Sousa, A. A. e Torres, G. L. (2007). Globally convergent optimal power flow by trust-region interiorpoint methods, IEEE PowerTech 200\%, Lausanne, Switzerland, pp. 1-6. 
Torres, G. L. e Carvalho Jr., M. A. (2006). On efficient implementation of interior-point based optimal power flows in rectangular coordinates, Proc. of the IEEE 2006 Power Systems Conference and Exposition, Atlanta, USA.

Torres, G. L. e Quintana, V. H. (1998). An interior point method for nonlinear optimal power flow using voltage rectangular coordinates, IEEE Trans. on Power Systems 13(4): 1211-1218.

Wang, H., Murillo-Sánchez, C. E., Zimmerman, R. D. e Thomas, R. J. (2007). On computational issues of market-based optimal power flow, IEEE Trans. on Power Systems 22(3): 1185-1193. 